OPEN ACCESS

Check for updates

\title{
Dietary fats and mortality among patients with type 2 diabetes: analysis in two population based cohort studies
}

\author{
Jingjing Jiao, ${ }^{1,2}$ Gang Liu, ${ }^{2}$ Hyun Joon Shin, ${ }^{4}$ Frank B Hu, ${ }^{2,5,6}$ Eric B Rimm, ${ }^{2,5,6}$ \\ Kathryn M Rexrode, ${ }^{7,8}$ JoAnn E Manson, ${ }^{5,6,7,8}$ Geng Zong, ${ }^{2,3^{*}}$ Qi Sun ${ }^{2,6^{*}}$
}

For numbered affiliations see end of the article.

${ }^{*}$ Contributed equally as senior authors

Correspondence to: Q Sun qisun@hsph.harvard.edu (ORCID 0000-0002-8480-1563) Additional material is published online only. To view please visit the journal online.

Cite this as: $B M J$ 2019;366:14009 http://dx.doi.org/10.1136/bmj.l4009

Accepted: 24 May 2019

\section{ABSTRACT}

OBJECTIVE

To assess the association of dietary fatty acids with cardiovascular disease mortality and total mortality among patients with type 2 diabetes.

DESIGN

Prospective, longitudinal cohort study.

SETTING

Health professionals in the United States.

\section{PARTICIPANTS}

11264 participants with type 2 diabetes in the Nurses' Health Study (1980-2014) and Health Professionals Follow-Up Study (1986-2014).

EXPOSURE

Dietary fat intake assessed using validated food frequency questionnaires and updated every two to four years.

\section{MAIN OUTCOME MEASURE}

Total and cardiovascular disease mortality during follow-up.

RESULTS

During follow-up, 2502 deaths including 646 deaths due to cardiovascular disease were documented. After multivariate adjustment, intake of polyunsaturated fatty acids (PUFAs) was associated with a lower cardiovascular disease mortality, compared with total carbohydrates: hazard ratios comparing the highest with the lowest quarter were 0.76 (95\% confidence interval 0.58 to 0.99 ; $P$ for trend $=0.03$ ) for total PUFAs, 0.69 (0.52 to 0.90; $P=0.007$ ) for

\section{WHAT IS ALREADY KNOWN ON THIS TOPIC}

Dietary guidelines for patients with diabetes recommend limiting trans fat intake and replacing saturated fats with unsaturated fats for maintaining good health These recommendations are largely based on findings in general populations Little is known about the associations of specific dietary fats with total and cardiovascular disease mortality among patients with diabetes who have altered metabolism of macronutrients

\section{WHAT THIS STUDY ADDS}

Among diabetes patients, higher dietary intake of total polyunsaturated fatty acids (PUFAs), a-linolenic acid, linoleic acid, and marine n-3 PUFAs is associated with lower total mortality

Intake of monounsaturated fats from animal sources is associated with higher total mortality

In an isocaloric model, theoretically replacing saturated fatty acids with PUFAs, especially linoleic acid, is associated with lower cardiovascular disease mortality

Increasing dietary PUFAs, especially linoleic acid and marine n-3 PUFAs, in replacement of saturated fatty acids, may facilitate long term survival among patients with diabetes marine n-3 PUFAs, 1.13 (0.85 to 1.51) for a-linolenic acid, and 0.75 (0.56 to 1.01) for linoleic acid. Inverse associations with total mortality were also observed for intakes of total PUFAs, n-3 PUFAs, and linoleic acid, whereas monounsaturated fatty acids of animal, but not plant, origin were associated with a higher total mortality. In models that examined the theoretical effects of substituting PUFAs for other fats, isocalorically replacing $2 \%$ of energy from saturated fatty acids with total PUFAs or linoleic acid was associated with $13 \%$ (hazard ratio $0.87,0.77$ to 0.99 ) or $15 \%(0.85,0.73$ to 0.99$)$ lower cardiovascular disease mortality, respectively. A $2 \%$ replacement of energy from saturated fatty acids with total PUFAs was associated with $12 \%$ (hazard ratio $0.88,0.83$ to 0.94 ) lower total mortality.

\section{CONCLUSIONS}

In patients with type 2 diabetes, higher intake of PUFAs, in comparison with carbohydrates or saturated fatty acids, is associated with lower total mortality and cardiovascular disease mortality. These findings highlight the important role of quality of dietary fat in the prevention of cardiovascular disease and total mortality among adults with type 2 diabetes.

\section{Introduction}

The number of people with type 2 diabetes is estimated to be 422 million globally and is projected to reach 642 million by $2040,{ }^{12}$ imposing a substantial disease toll and economic burden on patients and healthcare systems. Cardiovascular disease is the leading cause of deaths in adults with diabetes. ${ }^{3}$ Current dietary guidelines for the prevention and management of cardiovascular disease among patients with diabetes recommend limited intake of saturated fatty acids, trans fats, and cholesterol and higher consumption of foods abundant in omega-3 polyunsaturated fatty acids (PUFAs), ${ }^{4}$ such as fish, nuts, and seeds. However, these recommendations were largely based on findings from general populations. Owing to the altered metabolism of carbohydrates and fats, ${ }^{5}$ dyslipidemia, and the prothrombotic profile in patients with diabetes, ${ }^{6}$ whether the recommendations could be extrapolated to these patients has yet to be elucidated. The risk of cardiovascular disease among patients with type 2 diabetes is two to three times that among general populations, so the health effects of dietary fats, especially omega-3 PUFAs, in these patients needs to be clarified. ${ }^{7}$ A Mediterranean diet rich in monounsaturated or polyunsaturated fats has been shown to improve blood glucose and lipid control among people with diabetes, ${ }^{8-11}$ but the association between dietary fats and total mortality or mortality 
due to cardiovascular disease in such population remains unclear. ${ }^{12-15}$

To fill this knowledge gap, we assessed the associations of major dietary fats with cardiovascular disease mortality and total mortality among adults with diabetes in two large prospective cohort studies. We hypothesized that quality of fats determines their associations with total and cardiovascular disease mortality among patients with type 2 diabetes.

\section{Methods}

\section{Study population}

The Nurses' Health Study (NHS) is an ongoing cohort study consisting of 121701 female nurses aged 30-55 years at enrollment in $1976 .{ }^{16}$ The Health Professionals Follow-Up Study (HPFS) is a parallel cohort that started in 1986 and enrolled 51529 US male health professionals aged 40-75 years. ${ }^{17}$ Details of these two cohorts have been published elsewhere. ${ }^{17} 18$ Information on non-dietary lifestyle factors, medical history, and incident diseases was collected every two years through validated questionnaires. ${ }^{19}$ The cumulative response rate during follow-up was more than $90 \%$ in both cohorts. The return of completed questionnaires was considered to imply informed consent.

The analysis reported here included both prevalent cases of type 2 diabetes at study baseline (1980 for NHS or 1986 for HPFS when dietary data were first collected) and incident cases diagnosed during followup. We excluded participants who had cardiovascular disease or cancer at baseline or before diagnosis of type 2 diabetes, had implausible daily caloric intake $(<500$ or $>3500 \mathrm{kcal} /$ day for women; $<800$ or $>4200$ $\mathrm{kcal} /$ day for men), or had missing data of dietary fats at the entry of our analysis. The final sample included 1498 prevalent cases and 9766 incident cases (supplementary figure A).

\section{Ascertainment of type 2 diabetes}

Participants with a self reported diagnosis of diabetes were sent a validated supplementary questionnaire to obtain detailed information on the diabetes diagnosis, including date of diagnosis, symptoms, and treatment. Before 1997 cases of type 2 diabetes were diagnosed according to the original National Diabetes Data Group criteria if they met at least one of the following criteria: classic symptoms plus a fasting plasma glucose concentration of $140 \mathrm{mg} / \mathrm{dL}(7.8 \mathrm{mmol} / \mathrm{L})$ or above or a randomly measured plasma glucose concentration of $200 \mathrm{mg} / \mathrm{dL}$ (11.1 mmol/L) or above; at least two elevated plasma glucose concentrations on different occasions ( $\geq 140 \mathrm{mg} / \mathrm{dL}$ for fasting glucose or $\geq 200 \mathrm{mg}$ / $\mathrm{dL}$ for randomly measured glucose; $\geq 200 \mathrm{mg} / \mathrm{dL}$ for at least two hours after an oral glucose challenge); or treatment with hypoglycemic drugs (insulin or an oral hypoglycemic agent). ${ }^{20}$ From 1997 the fasting plasma glucose concentration for diagnosis of diabetes was lowered to $7.0 \mathrm{mmol} / \mathrm{L}$ or $126 \mathrm{mg} / \mathrm{dL}$ on the basis of the American Diabetes Association diagnostic criteria. ${ }^{21}$ In validation studies, $98 \%$ of cases of diabetes reported by questionnaire were reconfirmed by medical record review in the $\mathrm{NHS}^{22}$ and $97 \%$ of cases were reconfirmed in the HPFS. ${ }^{23}$ Given such high accuracy, we included all self reported cases of diabetes to increase the sample size.

\section{Dietary assessment}

Diet was assessed using validated semiquantitative food frequency questionnaires (FFQs) administered in 1980, 1984, 1986, and every four years thereafter in the NHS and every four years since 1986 in the HPFS, as described previously. ${ }^{2425}$ The overall validity and reliability of the semiquantitative FFQs have been reported previously. ${ }^{24}{ }^{25}$ Nutrient composition came from the Harvard University Food Composition Database, which is continuously updated to account for changes in nutrient contents and food processing. Fat intake was then calculated by multiplying the consumption frequency of each food item with a prespecified portion size (for example, one egg or one slice of bread) by the content of fats, taking account of the brand and type of fat used in preparation. Dietary fats were expressed as percentages of energy. We calculated cumulative averages of dietary variables over all valid assessments from the first dietary questionnaire after diagnosis of type 2 diabetes through the FFQ assessment made before the incidence of cardiovascular disease. In comparison with use of baseline dietary assessments only or updating the diet during follow-up using each FFQ assessment in isolation, using the cumulative averages helps to reduce within person variability and provide more stable estimate of long term diet, ${ }^{26}$ which is more biologically relevant to incidence of chronic disease. We stopped updating diet after a diagnosis of incident cardiovascular disease or cancer during study followup to minimize the possibility of reverse causation bias-patients with these conditions may adapt to a healthier diet but are still at a higher mortality risk than those without these conditions, and therefore a healthy diet may seem to be associated with early death if we continued to update their lifestyle/diet after the incidence of diseases.

In validation studies, the assessment of dietary fats was compared with that estimated by multiple one week diet records. Correlation coefficients between fat intakes assessed by the 1986 FFQ and diet records were 0.70 for saturated fatty acids, 0.69 for monounsaturated fatty acids (MUFAs), and 0.64 for PUFAs. $^{27}$

\section{Covariate assessments}

Information on lifestyle and other potential risk factors for mortality was assessed at baseline and updated during follow-up through biennial questionnaires, including age, ethnicity, weight, smoking status, alcohol intake, physical activity, family history of diabetes, family history of myocardial infarction, self reported hypertension and hypercholesterolemia, use of multivitamin, use of aspirin, and use of insulin and oral hypoglycemic drugs. Body mass index was 
calculated by dividing weight $(\mathrm{kg})$ by height squared $\left(\mathrm{m}^{2}\right)$. Physical activity was assessed as hours per week of moderate or vigorous activities (including brisk walking) that require the expenditure of at least 3 metabolic equivalents (METs) or more per hour. ${ }^{28}$

\section{Ascertainment of deaths}

We identified deaths by searching the National Death Index and through reports by next of kin or postal authorities. ${ }^{29}$ More than $98 \%$ of deaths were identified. $^{30}$ The cause of death was ascertained by physicians who were blinded to the risk profiles of the participants through review of death certificates and medical records. We used ICD-8 (international classification of diseases, 8th revision) and ICD-9 codes to classify cardiovascular mortality (ICD-8: 390.0458.9; ICD-9: 390.0-459.9) and cancer mortality (ICD8: 140.0-207.9; ICD-9: 140.0-208.9). ${ }^{31}$

\section{Statistical analysis}

We calculated person time from the return date of the first dietary questionnaire after diagnosis of type 2 diabetes to the time of death or the end of follow-up (June 30, 2014 for the NHS and January 30, 2014 for the HPFS), whichever was earlier. We applied Cox proportional hazards models to estimate hazard ratios and 95\% confidence intervals for the associations of different dietary fats with cardiovascular disease, cancer, and total mortality. To ensure a large enough number of deaths for analyses, we pooled participants from the two cohorts (no significant heterogeneity was found).

We built several isocaloric models to estimate relative risk of deaths when energy from saturated fats or carbohydrates was theoretically replaced by equivalent energy from another macronutrient. A key rationale of the substitution analysis is that, in the isocaloric setting, the reduction of one macronutrient as a percentage of total energy intake will be replaced by the same proportion of energy from another macronutrient, when total energy intake and intake of all other macronutrients are held constant. ${ }^{32}$ For example, to model the association with mortality when energy from carbohydrates is isocalorically replaced by PUFAs, we included PUFAs, total energy, monounsaturated fats, saturated fats, trans fats, and proteins in the multivariate model. In such a model, the $\beta$ coefficient of PUFAs bears interpretation as the theoretical effect of substituting PUFAs for the same amount of energy from carbohydrates. To make the risk estimates comparable for various macronutrient substitution analyses, we present results for $2 \%$ energy substitution, which is more realistic for macronutrients at low intake levels (or example, 1-2\% energy for trans fat). For specific PUFAs, such as arachidonic acid and marine n-3 PUFAs, with even lower intake levels (0.1-0.2\% energy), we used a smaller proportion of energy $(0.1 \%)$. Other covariates in the substitution analysis included age, sex, ethnicity, body mass index at diagnosis, smoking status, pack years of smoking, physical activity, alcohol consumption, family history of diabetes or myocardial infarction, self reported hypertension or hypercholesterolemia, duration of diabetes, multivitamin use, current aspirin use, and dietary cholesterol intake. We calculated tests for trend by modeling the median values of each category as a continuous variable.

In a secondary analysis, we further analyzed the associations for MUFAs from plant and animal origins separately. We did sensitivity analyses by excluding mortality that occurred within four years after diagnosis of diabetes, excluding prevalent type 2 diabetes, excluding participants with extreme body mass index $(<18.5$ or $>40.0)$, restricting the analysis to only confirmed cases of type 2 diabetes, or further adjusting for the use of lipid lowering or hypoglycemic drugs.

We used SAS 9.4 for statistical analyses. We considered two sided $\mathrm{P}$ values below 0.05 to be statistically significant.

\section{Patient and public involvement}

Participants were not involved in setting the research question or the outcome measures, nor were they involved in developing plans for recruitment, design, or implementation of the study. No participants were asked to advise on interpretation or writing up of the manuscript. The participants are updated on the study outcomes and developments through the study websites (www.nurseshealthstudy.org and https://www.hsph.harvard.edu/hpfs/index.html) and newsletters.

\section{Results}

\section{Population characteristics}

The sources of dietary fats, in particular trans fat, changed over time during follow-up, ${ }^{33}$ such that margarine was one of the primary sources of both trans fats and PUFAs in early, but not later, follow-up. Given this time trend, we show the characteristics of patients at the midpoint of follow-up. During a mean follow-up of 11 years (124362 person years), we documented 2502 deaths, including 646 deaths due to cardiovascular disease. At the midpoint of follow-up, women with higher PUFA intake tended to be older, had shorter duration of diabetes, and were less likely to have a family history of cancer than women with lower PUFA consumption. In addition, higher PUFA intake was correlated with lower intake of carbohydrates or trans fat but higher intake of MUFAs (table 1). Men with higher PUFA consumption tended to be younger and had higher pack years of smoking. Similarly, in men, higher PUFA intake was correlated with lower intake of carbohydrate and higher intakes of saturated fatty acids and MUFAs. Participants' characteristics according to saturated fatty acid and MUFA intake are shown in supplementary table A.

\section{Cardiovascular and total mortality}

We observed a significant inverse association of total PUFAs with cardiovascular disease mortality and total mortality after adjusting for age and sex (table 2). In 
a multivariate adjusted model that estimated mortality risk by substituting fats for carbohydrates, the association remained significant: hazard ratios across quarters of PUFA intake (from low to high) were 1.00, 0.99 (95\% confidence interval 0.80 to 1.23$), 0.85$ (0.67 to 1.08 ), and 0.76 (0.58 to 0.99 ) for cardiovascular disease mortality ( $P$ for trend=0.03) and 1.00, 0.86 (0.77 to 0.95 ), 0.83 (0.74 to 0.94 ), and 0.68 (0.60 to 0.78 ) for total mortality ( $P$ for trend $<0.001$ ). Intakes of saturated fatty acids and trans fats were positively associated with cardiovascular disease mortality in the age and sex adjusted model (model 1) but became non-significant after adjustment for other covariates (model 2; table 2).

Total MUFA intake was not significantly associated with either cardiovascular disease mortality or total mortality (model 2; table 2). Nevertheless, MUFAs from animal sources (mainly red meats and high fat dairy products) were positively associated with total mortality (hazard ratio comparing the highest versus lowest quarters $1.23,1.04$ to 1.45 ; $\mathrm{P}$ for trend $<0.001$; supplementary table $B$ ) and nonsignificantly associated with a higher cardiovascular disease mortality (hazard ratio 1.27, 0.92 to 1.75; $\mathrm{P}$ for trend=0.07). We found no significant associations with mortality for intake of plant derived MUFAs.

For specific PUFAs, intake of marine n-3 PUFAs was associated with a lower cardiovascular mortality after multivariate adjustment (hazard ratio comparing extreme quarters $0.69,0.52$ to 0.90 ; $\mathrm{P}$ for trend=0.007). The association between linoleic acid and cardiovascular disease mortality did not achieve statistical significance, with a hazard ratio of 0.75 (0.56 to 1.01; P for trend=0.06) (table 3). Intake of marine n-3 PUFAs and linoleic acid was associated with lower total mortality: hazard ratios comparing extreme quarters were 0.71 (0.62 to 0.82; $\mathrm{P}$ for trend $<0.001)$ and $0.81(0.69$ to 0.94 ; $\mathrm{P}$ for trend=0.008), respectively (table 3 ). Intake of $\alpha$-linolenic acid was non-significantly associated with lower total mortality (hazard ratio $0.88,0.76$ to 1.02; P for trend=0.04).

\section{Cancer mortality}

We observed no significant associations of saturated fatty acid and MUFA intake with cancer mortality (supplementary table C). As shown in supplementary table $\mathrm{D}$, participants in the highest quarter of marine n-3 PUFA intake had a 28\% (hazard ratio 0.72, 0.53 to 0.99 ) lower risk of cancer mortality, compared with those in the lowest quarter. Intake of $\alpha$-linolenic acid was inversely associated with cancer mortality, but the hazard ratio comparing extreme quarters did not achieve statistical significance $(0.75,0.53$ to 1.05 ; $\mathrm{P}$ for trend=0.04).

\section{Substitution for saturated fatty acids}

Figure 1 shows mortality risk by isocaloric replacement of saturated fatty acids with other fats. Mortality due to cardiovascular disease was $13 \%$ (hazard ratio 0.87 , 0.77 to 0.99 ) lower when $2 \%$ energy from saturated fatty acids was isocalorically replaced by total PUFAs and $15 \%(0.85,0.73$ to 0.99$)$ lower when it was replaced by linoleic acid. Similarly, replacing $2 \%$ energy from saturated fatty acids with total PUFAs was associated with $12 \%$ (hazard ratio $0.88,0.83$ to 0.94 ) lower total mortality. We found no significant changes in total mortality (hazard ratio $0.99,0.94$ to 1.05) or cardiovascular disease mortality $(0.99,0.88$ to 1.11 ) when replacing $2 \%$ energy from saturated fatty acids with MUFAs. Replacing saturated fatty acids with PUFAs was not associated with cancer mortality (supplementary figure B).

\section{Sensitivity analyses}

The associations of specific dietary fats with total, cardiovascular disease, and cancer mortality did not change materially after exclusion of participants with extreme body mass index or further adjustment for the use of hypoglycemic drugs (supplementary table E). Excluding deaths that occurred within four years after diagnosis of type 2 diabetes slightly strengthened the results. We observed similar results when we further adjusted for the use of lipid lowering drugs or restricted the analysis to only incident cases of diabetes. Restricting the analysis to only confirmed cases of type 2 diabetes slightly attenuated the associations.

\section{Discussion}

In two cohorts of US men and women, we showed an inverse association of dietary PUFAs, including linoleic acid and marine n-3 PUFAs, with mortality due to cardiovascular disease or total mortality among patients with type 2 diabetes. In addition, animal derived MUFAs were associated with higher total mortality when compared with dietary carbohydrates.

\section{Comparison with other studies and possible explanations}

To our knowledge, this is the first prospective analysis that investigated the associations between specific dietary fats and cardiovascular disease mortality among people with type 2 diabetes. Our findings on PUFAs are largely ascribed to linoleic acid, which is the most abundant PUFA in the diet. ${ }^{4}$ Similarly, a recent analysis in the NHS and HPFS showed a significant inverse association of dietary linoleic acid with total and cardiovascular disease mortality among participants who were free of major chronic diseases, including diabetes, at baseline. ${ }^{34}$ However, these findings are seemingly contradictory to evidence from the Sydney Diet Heart Study showing that higher linoleic acid intake led to increased mortality. ${ }^{35}$ We note that safflower oil and margarines were used as the food source of linoleic acid in this intervention, so the observed effect may be at least partially ascribed to the high trans fat contents of margarines at that time. ${ }^{36}$ In contrast, a recent meta-analysis of four randomized controlled trials showed that substituting PUFAs for saturated fatty acids reduced the risk of coronary heart disease by approximately 30\%. ${ }^{37}$ Our findings are also in line with a 30 week intervention study that 


\begin{tabular}{|c|c|c|c|c|}
\hline \multirow[b]{2}{*}{ Characteristics } & \multicolumn{4}{|c|}{ Quarters of polyunsaturated fat intake (\% energy) } \\
\hline & $1(\leq 5.22)$ & $2(5.22-6.21)$ & $3(6.21-7.41)$ & $4(\geq 7.41)$ \\
\hline Nurses' Health Study $(n=9053)$ & $(n=2358)$ & $(n=2275)$ & $(n=2224)$ & $(n=2196)$ \\
\hline Mean (SD) age, years & $69.7(10.6)$ & $72.6(8.4)$ & $73.1(7.3)$ & $73.6(7.2)$ \\
\hline White ethnicity & $2200(93.3)$ & $2230(98.0)$ & $2184(98.2)$ & $2154(98.1)$ \\
\hline Age adjusted mean (SD) body mass index & $29.4(6.8)$ & $29.3(7.1)$ & $30.3(6.4)$ & $28.9(5.5)$ \\
\hline Age adjusted mean (SD) alcohol consumption, g/day & $3.8(8.9)$ & $3.3(6.7)$ & $3.5(7.9)$ & $3.7(7.7)$ \\
\hline Age adjusted mean (SD) physical activity, h/week & $1.0(2.2)$ & $1.9(3.4)$ & $1.2(2.8)$ & $1.8(3.2)$ \\
\hline Current smoker & $391(16.6)$ & $141(6.2)$ & $176(7.9)$ & $182(8.3)$ \\
\hline Age adjusted mean (SD) pack years' smoking & $336.6(444.7)$ & $434.2(471.2)$ & $407.0(461.3)$ & $509.7(471.8)$ \\
\hline Multivitamin use & $1139(48.3)$ & $1240(54.5)$ & $1305(58.7)$ & $1269(57.8)$ \\
\hline Family history of diabetes & $1113(47.2)$ & $858(37.7)$ & $832(37.4)$ & $898(40.9)$ \\
\hline Family history of myocardial infarction & $729(30.9)$ & $669(29.4)$ & $483(21.7)$ & $525(23.9)$ \\
\hline Family history of cancer & $394(16.7)$ & $355(15.6)$ & $282(12.7)$ & $184(8.4)$ \\
\hline Aspirin use & $1101(46.7)$ & $1279(56.2)$ & $1285(57.8)$ & $1228(55.9)$ \\
\hline Hypoglycemic drug use & $1205(51.1)$ & $1413(62.1)$ & $1466(65.9)$ & $1377(62.7)$ \\
\hline Age adjusted mean (SD) duration of diabetes, years & $3.5(2.1)$ & $3.5(2.3)$ & $3.4(1.8)$ & $3.2(1.5)$ \\
\hline Hypercholesterolemia & $1743(73.9)$ & $1859(81.7)$ & $1750(78.7)$ & $1810(82.4)$ \\
\hline Hypertension & $2122(90.0)$ & $2073(91.1)$ & $2051(92.2)$ & $1957(89.1)$ \\
\hline Age adjusted mean (SD) total energy intake, kcal/day & $1561.9(527.4)$ & $1618.5(471.4)$ & $1612.0(451.1)$ & $1603.6(520.4)$ \\
\hline \multicolumn{5}{|l|}{ Age adjusted mean (SD) intakes, \% energy: } \\
\hline Carbohydrates & $53.8(9.2)$ & $50.9(8.9)$ & $49.8(6.8)$ & $46.2(6.4)$ \\
\hline Protein & $18.8(3.8)$ & $18.6(3.5)$ & $18.1(2.9)$ & $17.9(3.2)$ \\
\hline Total fats & $27.4(6.5)$ & $31.0(6.0)$ & $32.6(5.2)$ & $36.4(5.5)$ \\
\hline Total PUFAs & $4.4(0.7)$ & $5.8(0.3)$ & $6.8(0.3)$ & $9.4(2.2)$ \\
\hline $\mathrm{n}-3$ PUFAs & $0.6(0.2)$ & $0.7(0.2)$ & $0.9(0.2)$ & $1.2(0.5)$ \\
\hline a-linolenic acid & $0.5(0.1)$ & $0.6(0.1)$ & $0.7(0.2)$ & $1.0(0.5)$ \\
\hline Marine $\mathrm{n}$-3 PUFAs & $0.1(0.1)$ & $0.2(0.2)$ & $0.2(0.2)$ & $0.2(0.2)$ \\
\hline $\mathrm{n}-6$ PUFAs & $3.9(0.9)$ & $5.1(0.7)$ & $6.1(0.6)$ & $8.3(1.9)$ \\
\hline Linoleic acid & $3.8(0.9)$ & $5.0(0.7)$ & $6.0(0.6)$ & $8.2(1.9)$ \\
\hline Arachidonic acid & $0.1(0.0)$ & $0.1(0.0)$ & $0.1(0.0)$ & $0.1(0.0)$ \\
\hline Saturated fatty acids & $10.3(3.4)$ & $10.7(3.3$ & $10.9(2.8)$ & $10.7(2.6)$ \\
\hline Monounsaturated fatty acids & $10.1(3.0)$ & $11.9(3.4)$ & $12.0(2.8)$ & $13.5(3.0)$ \\
\hline Trans fats & $1.3(0.5)$ & $1.2(0.7)$ & $1.1(0.6)$ & $1.0(0.7)$ \\
\hline Health Professionals Follow-up Study $(n=2211)$ & $(n=476)$ & $(n=539)$ & $(n=582)$ & $(n=614)$ \\
\hline Mean (SD) age, years & $75.0(9.9)$ & $74.1(9.3)$ & $72.1(8.0)$ & $71.8(6.4)$ \\
\hline White ethnicity & $419(88.0)$ & $497(92.3)$ & $539(92.6)$ & $573(93.3)$ \\
\hline Age adjusted mean (SD) body mass index & $26.9(3.9)$ & $29.3(4.7)$ & $27.9(4.5)$ & $27.9(4.1)$ \\
\hline Age adjusted mean (SD) alcohol consumption, g/day & $15.3(19.0)$ & $13.2(15.0)$ & $8.4(8.5)$ & $10.0(11.8)$ \\
\hline Age adjusted mean (SD) physical activity, h/week & $2.4(3.2)$ & $5.4(17.2)$ & $3.5(5.2)$ & $6.1(10.0)$ \\
\hline Current smoker & $16(3.3)$ & $9(1.6)$ & $53(9.1)$ & $24(3.9)$ \\
\hline Age adjusted mean (SD) pack years' smoking & $193.5(327.8)$ & $265.5(410.4)$ & $308.1(410.3)$ & $328.1(423.7)$ \\
\hline Multivitamin use & $264(55.4)$ & $294(54.6)$ & $342(58.8)$ & $355(57.8)$ \\
\hline Family history of diabetes & $246(51.7)$ & $160(29.6)$ & $100(17.2)$ & $269(43.8)$ \\
\hline Family history of myocardial infarction & $218(45.7)$ & $176(32.6)$ & $158(27.1)$ & $193(31.5)$ \\
\hline Family history of cancer & $99(20.9)$ & $209(38.7)$ & $250(43.0)$ & $226(36.8)$ \\
\hline Aspirin use & $306(64.2)$ & $309(57.4)$ & $332(57.0)$ & $371(60.5)$ \\
\hline Hypoglycemic drug use & $153(32.2)$ & $156(29.0)$ & $177(30.4)$ & $279(45.4)$ \\
\hline Age adjusted mean (SD) duration of diabetes, years & $3.0(0.8)$ & $3.2(0.8)$ & $3.1(1.0)$ & $3.2(1.5)$ \\
\hline Hypercholesterolemia & $317(66.7)$ & $434(80.6)$ & $442(76.0)$ & $432(70.4)$ \\
\hline Hypertension & $361(75.9)$ & $470(87.2)$ & $443(76.1)$ & $468(76.2)$ \\
\hline Age adjusted mean (SD) total energy intake, kcal/day & $1763.8(447.3)$ & $1908.2(478.1)$ & $1778.6(559.0)$ & $2141.4(739.7)$ \\
\hline \multicolumn{5}{|l|}{ Age adjusted mean (SD) intakes, \% energy: } \\
\hline Carbohydrates & $51.5(8.0)$ & $47.8(6.1)$ & $47.2(7.0)$ & $43.0(7.0)$ \\
\hline Protein & $18.0(3.3)$ & $18.4(4.3)$ & $18.4(3.2)$ & $18.5(2.9)$ \\
\hline Total fats & $27.1(4.8)$ & $30.5(4.8)$ & $32.9(5.3)$ & $37.7(6.2)$ \\
\hline Total PUFAs & $4.5(0.5)$ & $5.8(0.3)$ & $6.8(0.3)$ & $9.6(2.6)$ \\
\hline $\mathrm{n}-3$ PUFAs & $0.6(0.2)$ & $0.7(0.3)$ & $0.8(0.2)$ & $1.3(0.6)$ \\
\hline a-linolenic acid & $0.4(0.1)$ & $0.5(0.2)$ & $0.6(0.2)$ & $1.0(0.5)$ \\
\hline Marine $n-3$ PUFAs & $0.2(0.1)$ & $0.2(0.2)$ & $0.2(0.1)$ & $0.3(0.2)$ \\
\hline n-6 PUFAs & $4.2(0.6)$ & $5.1(0.5)$ & $6.2(0.5)$ & $8.5(2.1)$ \\
\hline Linoleic acid & $4.1(0.6)$ & $5.0(0.5)$ & $6.1(0.5)$ & $8.4(2.1)$ \\
\hline Arachidonic acid & $0.1(0.0)$ & $0.1(0.0)$ & $0.1(0.0)$ & $0.1(0.0)$ \\
\hline Saturated fatty acids & $9.8(2.4)$ & $10.3(2.8)$ & $10.4(2.6)$ & $10.5(2.3)$ \\
\hline Monounsaturated fatty acids & $10.2(2.1)$ & $11.7(2.1)$ & $12.7(2.7)$ & $14.5(3.4)$ \\
\hline Trans fats & $1.1(0.5)$ & $1.4(0.7)$ & $1.2(0.6)$ & $1.1(0.8)$ \\
\hline
\end{tabular}




\begin{tabular}{|c|c|c|c|c|c|}
\hline & \multicolumn{4}{|c|}{ Quarters of fatty acids intake } & \multirow{2}{*}{$\begin{array}{l}\mathrm{P} \text { for } \\
\text { trend }\end{array}$} \\
\hline & 1 & 2 & 3 & 4 & \\
\hline \multicolumn{6}{|l|}{ Polyunsaturated fat intake } \\
\hline Median (range) \% energy & $4.48(\leq 5.06)$ & $5.50(5.07-5.97)$ & $6.39(5.98-7.06)$ & $7.95(\geq 7.07)$ & \\
\hline \multicolumn{6}{|c|}{ Cardiovascular disease mortality: } \\
\hline No of cases/person years & $198 / 31335$ & $176 / 31490$ & $147 / 31569$ & $125 / 31600$ & \\
\hline Model $1^{\star}$ & 1.00 & 0.98 (0.79 to 1.20$)$ & $0.84(0.67$ to 1.04$)$ & $0.74(0.59$ to 0.93$)$ & 0.004 \\
\hline Model $2 \dagger$ & 1.00 & 0.99 (0.80 to 1.23$)$ & 0.85 (0.67 to 1.08$)$ & 0.76 (0.58 to 0.99) & 0.03 \\
\hline \multicolumn{6}{|l|}{ Total mortality: } \\
\hline No of cases/person years & $855 / 30754$ & $627 / 31082$ & $575 / 31189$ & $445 / 31336$ & \\
\hline Model $1^{\star}$ & 1.00 & $0.81(0.73$ to 0.90$)$ & $0.78(0.70$ to 0.86$)$ & $0.61(0.55$ to 0.69$)$ & $<0.001$ \\
\hline Model $2 \dagger$ & 1.00 & $0.86(0.77$ to 0.95$)$ & $0.83(0.74$ to 0.94$)$ & 0.68 (0.60 to 0.78$)$ & $<0.001$ \\
\hline \multicolumn{6}{|l|}{ Monounsaturated fatty acids } \\
\hline Median (range) \% energy & $9.51(\leq 10.77)$ & $11.66(10.78-12.61)$ & $13.37(12.62-14.64)$ & $16.01(\geq 14.65)$ & \\
\hline \multicolumn{6}{|c|}{ Cardiovascular disease mortality: } \\
\hline No of cases/person years & $160 / 31453$ & $163 / 31524$ & $151 / 31559$ & $172 / 31458$ & \\
\hline Model $1^{\star}$ & 1.00 & $1.08(0.87$ to 1.35$)$ & 1.05 (0.84 to 1.32$)$ & $1.31(1.05$ to 1.64$)$ & 0.02 \\
\hline Model $2 \dagger$ & 1.00 & $0.96(0.74$ to 1.24$)$ & $0.85(0.63$ to 1.15$)$ & 0.99 (0.70 to 1.39) & 0.97 \\
\hline \multicolumn{6}{|l|}{ Total mortality: } \\
\hline No of cases/person years & $694 / 30975$ & $635 / 31088$ & $580 / 31203$ & $593 / 31096$ & \\
\hline Model $1^{*}$ & 1.00 & 1.01 (0.90 to 1.12$)$ & 0.97 (0.87 to 1.09$)$ & $1.08(0.97$ to 1.21$)$ & 0.23 \\
\hline Model $2 \dagger$ & 1.00 & $0.93(0.82$ to 1.05$)$ & $0.83(0.72$ to 0.96$)$ & $0.90(0.76$ to 1.06$)$ & 0.21 \\
\hline \multicolumn{6}{|l|}{ Saturated fatty acids } \\
\hline Median (range) \% energy & $8.03(\leq 9.28)$ & $10.03(9.29-11.09)$ & $11.69(11.10-13.18)$ & $14.34(\geq 13.19)$ & \\
\hline \multicolumn{6}{|c|}{ Cardiovascular disease mortality: } \\
\hline No of cases/person years & $138 / 31501$ & $166 / 31535$ & $154 / 31558$ & $188 / 31400$ & \\
\hline Model $1^{\star}$ & 1.00 & $1.30(1.03$ to 1.64$)$ & 1.26 (1.00 to 1.59$)$ & 1.71 (1.37 to 2.14$)$ & $<0.00$ \\
\hline Model $2+$ & 1.00 & 1.14 (0.88 to 1.48$)$ & $0.98(0.73$ to 1.33$)$ & $1.13(0.80$ to 1.59$)$ & 0.62 \\
\hline \multicolumn{6}{|l|}{ Total mortality: } \\
\hline No of cases/person years & $594 / 31087$ & $628 / 31103$ & $613 / 31184$ & $667 / 30988$ & \\
\hline Model $1^{*}$ & 1.00 & $1.14(1.02$ to 1.28$)$ & 1.21 (1.08 to 1.36$)$ & $1.39(1.24$ to 1.56$)$ & $<0.001$ \\
\hline Model $2 \dagger$ & 1.00 & 1.05 (0.92 to 1.19$)$ & 1.03 (0.89 to 1.19$)$ & $1.00(0.85$ to 1.19$)$ & 0.88 \\
\hline \multicolumn{6}{|l|}{ Trans fatty acids } \\
\hline Median (range) \% energy & $0.94(\leq 1.16)$ & $1.37(1.17-1.53)$ & $1.71(1.54-1.95)$ & $2.24(\geq 1.96)$ & \\
\hline \multicolumn{6}{|c|}{ Cardiovascular disease mortality: } \\
\hline No of cases/person year & $140 / 31499$ & $149 / 31542$ & $165 / 31546$ & $192 / 31407$ & \\
\hline Model ${ }^{\star}$ & 1.00 & 1.10 (0.87 to 1.39$)$ & $1.25(0.99$ to 1.57$)$ & 1.49 (1.19 to 1.86$)$ & $<0.001$ \\
\hline Model $2 \dagger$ & 1.00 & $0.97(0.76$ to 1.25$)$ & $1.03(0.80$ to 1.34$)$ & $1.13(0.86$ to 1.50$)$ & 0.28 \\
\hline \multicolumn{6}{|l|}{ Total mortality: } \\
\hline No of cases/person years & $558 / 31131$ & $574 / 31176$ & $638 / 31115$ & $732 / 30940$ & \\
\hline Model $1^{\star}$ & 1.00 & 1.06 (0.94 to 1.19) & $1.21(1.08$ to 1.36$)$ & $1.42(1.27$ to 1.58$)$ & $<0.001$ \\
\hline Model $2 \dagger$ & 1.00 & $0.93(0.82$ to 1.06$)$ & $1.01(0.88$ to 1.15$)$ & $1.08(0.94$ to 1.25$)$ & 0.11 \\
\hline \multicolumn{6}{|c|}{$\begin{array}{l}\text { *Adjusted for age (in months), sex, and survey period. } \\
\text { tFurther adjusted for ethnicity (white, others), body mass index at diagnosis ( }<23.0,23.0-24.9,25.0-29.9,30.0-34.9, \geq 35.0) \text {, physical activity (0-0.4, } \\
0.5-1.9,2.0-3.4,3.5-5.4, \geq 5.5 \mathrm{~h} / \text { week), smoking status (never, past, current } 1-14 \text { cigarettes/d, current } \geq 15 \text { cigarettes/d), smoking pack years }(0,<20 \text {, } \\
\geq 20 \text { pack years), alcohol consumption (0, 0.1-4.9, 5.0-14.9, 15.0-29.9, } \geq 30.0 \mathrm{~g} / \mathrm{d} \text { ), multivitamin use (yes, no), current aspirin use (yes, no), family } \\
\text { history of myocardial infarction (yes, no), family history of diabetes (yes, no), history of hypercholesterolemia (yes, no), history of hypertension (yes, no), } \\
\text { duration of diabetes ( }<5,5-10,>10 \text { years), total energy intake (quarters), dietary cholesterol (quarters), and percentage of energy from dietary protein } \\
\text { and remaining fatty acids where appropriate (polyunsaturated fatty acids (PUFAs), monounsaturated fatty acids, trans fats, linoleic acid, arachidonic acid, } \\
\text { a-linolenic acid, and marine n-3 PUFAs; all continuous variables). In this model, the } \beta \text { coefficients of dietary fats bear meaning of effects of isocalorically } \\
\text { substituting for total carbohydrates. }\end{array}$} \\
\hline
\end{tabular}

showed that a diet enriched with linoleic acid (10.9\% of energy) reduced concentrations of total cholesterol and low density lipoprotein cholesterol among people with type 2 diabetes. ${ }^{38}$ Collectively, these data suggest that intake of n-6 PUFAs may exert beneficial effects on cardiovascular health among people with and without type 2 diabetes.

Potential cardiovascular benefits of marine $n-3$ PUFAs have been reported in numerous cohort studies, ${ }^{39} 40$ although a recent meta-analysis did not show protective effects of marine $n-3$ supplements on cardiovascular outcomes, including cardiac death. ${ }^{41}$ This inconsistency may be due to high baseline dietary marine n-3 PUFA intake, few cardiac deaths, revascularization therapy, and use of statins and other lipid lowering drugs that may mask the effects of a low to moderate dose of fish oil supplementation in patients with cardiovascular disease. ${ }^{42}$ In the recent VITAL trial, marine $\mathrm{n}-3$ fatty acid supplementation significantly lowered the risk of cardiovascular disease among participants who had a lower intake of these fatty acids at baseline. ${ }^{43}$ Moreover, in the REDUCE-IT trial, which used a high dose ( $4 \mathrm{~g} /$ day) of pure eicosapentaenoic acid supplementation, the risk of developing cardiovascular disease was substantially reduced. ${ }^{44}$ Evidence specifically pertinent to patients with diabetes is relatively sparse. Clinical trials looking at intermediate cardiovascular 


\begin{tabular}{|c|c|c|c|c|c|}
\hline & \multicolumn{4}{|c|}{ Quarters of fatty acids intake } & \multirow{2}{*}{$\begin{array}{l}P \text { for } \\
\text { trend }\end{array}$} \\
\hline & 1 & 2 & 3 & 4 & \\
\hline \multicolumn{6}{|l|}{ Marine $\mathrm{n}-3$ PUFAs* } \\
\hline Median (range) \% energy & $0.03(\leq 0.05)$ & $0.07(0.06-0.09)$ & $0.13(0.10-0.17)$ & $0.25(\geq 0.17)$ & \\
\hline \multicolumn{6}{|c|}{ Cardiovascular disease mortality: } \\
\hline No of cases/person years & $198 / 31217$ & $169 / 31439$ & $166 / 31618$ & $113 / 31721$ & \\
\hline Model $1 \dagger$ & 1.00 & $0.83(0.67$ to 1.02$)$ & $0.78(0.63$ to 0.97$)$ & $0.54(0.42$ to 0.68$)$ & $<0.001$ \\
\hline Model $2 \ddagger$ & 1.00 & $0.92(0.74$ to 1.14$)$ & $0.91(0.72$ to 1.14$)$ & $0.69(0.52$ to 0.90$)$ & 0.007 \\
\hline \multicolumn{6}{|l|}{ Total mortality: } \\
\hline No of cases/person years & $791 / 30674$ & $703 / 30974$ & $606 / 31239$ & $402 / 31475$ & \\
\hline Model $1 \dagger$ & 1.00 & $0.89(0.80$ to 0.98$)$ & $0.77(0.69$ to 0.85$)$ & $0.52(0.46$ to 0.59$)$ & $<0.001$ \\
\hline Model 2‡ & 1.00 & $0.99(0.89$ to 1.10$)$ & 0.91 (0.81 to 1.03$)$ & $0.71(0.62$ to 0.82$)$ & $<0.001$ \\
\hline \multicolumn{6}{|l|}{ a-linolenic acid } \\
\hline Median (range) \% energy & $0.40(\leq 0.45)$ & $0.49(0.46-0.54)$ & $0.58(0.55-0.65)$ & $0.76(\geq 0.66)$ & \\
\hline \multicolumn{6}{|c|}{ Cardiovascular disease mortality: } \\
\hline No of cases/person years & $195 / 31291$ & $168 / 31509$ & $142 / 31578$ & $141 / 31616$ & \\
\hline Model 1† & 1.00 & $0.96(0.78$ to 1.18$)$ & 0.83 (0.66 to 1.03$)$ & $0.84(0.67$ to 1.04$)$ & 0.07 \\
\hline Model $2 \ddagger$ & 1.00 & 1.06 (0.85 to 1.32$)$ & $0.98(0.76$ to 1.26$)$ & $1.13(0.85$ to 1.51$)$ & 0.44 \\
\hline \multicolumn{6}{|l|}{ Total mortality: } \\
\hline No of cases/person years & $827 / 30715$ & $680 / 31066$ & $510 / 31253$ & $485 / 31328$ & \\
\hline Model 1† & 1.00 & 0.89 (0.80 to 0.99$)$ & 0.69 (0.62 to 0.77$)$ & 0.65 (0.58 to 0.73$)$ & $<0.001$ \\
\hline Model $2 \ddagger$ & 1.00 & 0.98 (0.87 to 1.09$)$ & $0.80(0.70$ to 0.91$)$ & $0.88(0.76$ to 1.02$)$ & 0.04 \\
\hline \multicolumn{6}{|l|}{ Linoleic acid } \\
\hline Median (range) \% energy & $3.65(\leq 4.21)$ & $4.72(4.22-5.13)$ & $5.55(5.14-6.16)$ & $7.03(\geq 6.17)$ & \\
\hline \multicolumn{6}{|c|}{ Cardiovascular disease mortality: } \\
\hline No of cases/person years & $209 / 31299$ & $161 / 31509$ & $149 / 31545$ & $127 / 31641$ & \\
\hline Model 1† & 1.00 & 0.81 (0.66 to 1.00$)$ & $0.76(0.61$ to 0.94$)$ & $0.67(0.53$ to 0.84$)$ & $<0.001$ \\
\hline Model $2 \ddagger$ & 1.00 & $0.86(0.69$ to 1.07$)$ & $0.81(0.63$ to 1.05$)$ & $0.75(0.56$ to 1.01$)$ & 0.06 \\
\hline \multicolumn{6}{|l|}{ Total mortality: } \\
\hline No of cases/person years & $848 / 30730$ & $623 / 31096$ & $575 / 31163$ & $456 / 31373$ & \\
\hline Model $1 \dagger$ & 1.00 & $0.80(0.72$ to 0.88$)$ & $0.76(0.68$ to 0.84$)$ & $0.62(0.55$ to 0.69$)$ & $<0.001$ \\
\hline Model $2 \ddagger$ & 1.00 & $0.89(0.80$ to 1.00$)$ & $0.90(0.80$ to 1.02$)$ & 0.81 (0.69 to 0.94$)$ & 0.008 \\
\hline \multicolumn{6}{|l|}{ Arachidonic acid } \\
\hline Median (range) \% energy & $0.05(\leq 0.06)$ & $0.07(0.07-0.08)$ & $0.09(0.09-0.10)$ & $0.12(\geq 0.11)$ & \\
\hline \multicolumn{6}{|c|}{ Cardiovascular disease mortality: } \\
\hline No of cases/person years & $168 / 31429$ & $142 / 31531$ & $165 / 31533$ & $171 / 31502$ & \\
\hline Model $1 \dagger$ & 1.00 & $0.91(0.73$ to 1.14$)$ & $1.13(0.91$ to 1.41$)$ & $1.16(0.93$ to 1.45$)$ & 0.07 \\
\hline Model 2‡ & 1.00 & 0.83 (0.65 to 1.07$)$ & 1.05 (0.80 to 1.39$)$ & $1.00(0.71$ to 1.39$)$ & 0.73 \\
\hline \multicolumn{6}{|l|}{ Total mortality: } \\
\hline No of cases/person years & $711 / 30946$ & $599 / 31139$ & $621 / 31132$ & $571 / 31144$ & \\
\hline Model 1t & 1.00 & $0.93(0.83$ to 1.04$)$ & 1.01 (0.91 to 1.13$)$ & $0.98(0.88$ to 1.10$)$ & 0.90 \\
\hline Model $2 \ddagger$ & 1.00 & 0.91 (0.80 to 1.02$)$ & 1.05 (0.91 to 1.21$)$ & 1.01 (0.85 to 1.19$)$ & 0.64 \\
\hline \multicolumn{6}{|c|}{$\begin{array}{l}\text { *Sum of eicosapentaenoic acid and docosahexaenoic acid. } \\
\text { TAdjusted for age (in months), sex, and survey period. } \\
\text { FFurther adjusted for ethnicity (white, others), body mass index at diagnosis ( }<23.0,23.0-24.9,25.0-29.9,30.0-34.9, \geq 35.0) \text {, physical activity (0-0.4, } \\
0.5-1.9,2.0-3.4,3.5-5.4, \geq 5.5 \mathrm{~h} / \text { week), smoking status (never, past, current } 1-14 \text { cigarettes/d, current } \geq 15 \text { cigarettes/d), smoking pack years }(0,<20, \\
\geq 20 \text { pack years), alcohol consumption (0, } 0.1-4.9,5.0-14.9,15.0-29.9, \geq 30.0 \mathrm{~g} / \mathrm{d}) \text {, multivitamin use (yes, no), current aspirin use (yes, no), family } \\
\text { history of myocardial infarction (yes, no), family history of diabetes (yes, no), history of hypercholesterolemia (yes, no), history of hypertension (yes, no), } \\
\text { duration of diabetes ( }<5,5-10,>10 \text { years), total energy intake (quarters), dietary cholesterol (quarters), and percentage of energy from dietary protein } \\
\text { and remaining fatty acids where appropriate (polyunsaturated fatty acids (PUFAs), monounsaturated fatty acids, trans fats, linoleic acid, arachidonic acid, } \\
\text { a-linolenic acid, and marine n-3 PUFAs; all continuous variables). In this model, the } \beta \text { coefficients of dietary fats bear meaning of effects of isocalorically } \\
\text { substituting for total carbohydrates. }\end{array}$} \\
\hline
\end{tabular}

disease outcomes among adults with diabetes have consistently reported that marine n-3 supplements could lower triglyceride concentrations, ${ }^{45}$ improve arterial blood flow, and ameliorate inflammatory conditions that predispose cardiovascular disease events. $^{46} 47$ Meanwhile, a secondary analysis of the JELIS study found that eicosapentaenoic acid supplementation ( $1.8 \mathrm{~g} /$ day) for 4.6 years led to a $22 \%$ reduction in risk of coronary artery disease among 4565 adults with type 2 diabetes or impaired glucose metabolism, ${ }^{48}$ but this effect was not observed in another randomized controlled trial ( $0.9 \mathrm{~g} / \mathrm{day})$ with a larger sample size $(n=12536)$ and longer follow-up
(6.2 years). ${ }^{49}$ In the latest trial of 15480 patients with diabetes, although daily supplementation with $0.84 \mathrm{~g}$ marine n-3 PUFAs for 7.4 years did not significantly lower the risk of major vascular events, including non-fatal myocardial infarction or stroke, transient ischemic attack, and vascular death, the vascular deaths were fewer in the marine n-3 fatty acid group than in the control group (rate ratio 0.82, 95\% confidence interval 0.68 to 0.98$){ }^{7}$

The morbidity and mortality of some cancers, such as endometrial, breast, and colorectal cancer is elevated in people with type 2 diabetes. ${ }^{50}$ The inverse association of marine n-3 PUFAs and $\alpha$-linolenic acid 


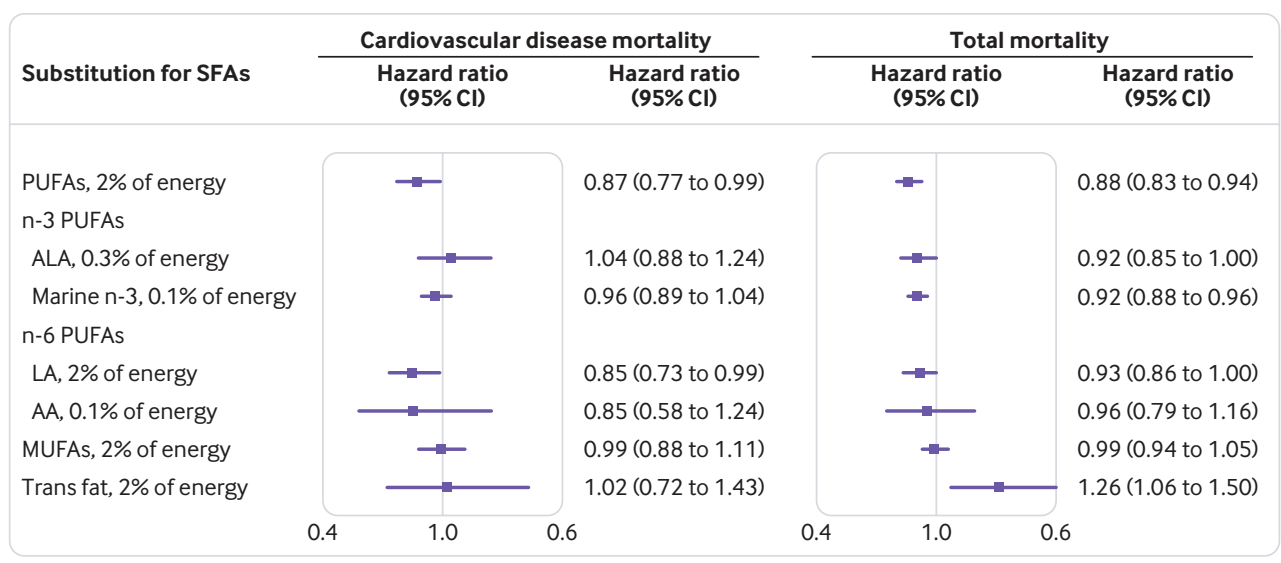

Fig 1 | Multivariate adjusted hazard ratios of cardiovascular disease mortality and total mortality by isocaloric substitution of specific types of dietary fats for saturated fatty acids (SFAs). Model was adjusted for age (in months), sex, survey period, white ethnicity (yes, no), body mass index at diagnosis ( $23.0,23.0-24.9,25.0-29.9,30.0-34.9$, $\geq 35.0$ ), physical activity $(0-0.4,0.5-1.9,2.0-3.4,3.5-5.4, \geq 5.5 \mathrm{~h} /$ week), smoking status (never, past, current 1-14 cigarettes/d, current $\geq 15$ cigarettes/d), smoking pack years $(0,<20, \geq 20)$, alcohol consumption $(0,0.1-4.9,5.0-14.9$, 15.0-29.9, $\geq 30.0 \mathrm{~g} / \mathrm{d}$ ), multivitamin use (yes, no), current aspirin use (yes, no), family history of myocardial infarction (yes, no), family history of diabetes (yes, no), history of hypercholesterolemia (yes, no), history of hypertension (yes, no), diabetes duration ( $\langle 5,5-10,>10$ years), total energy intake (quarters), dietary cholesterol (quarters), and percentage of energy from dietary protein, carbohydrates, and remaining fatty acids where appropriate (polyunsaturated fatty acids (PUFAs), monounsaturated fatty acids (MUFAs), trans fats, linoleic acid (LA), arachidonic acid (AA), a-linolenic acid (ALA), and marine n-3 PUFAs, all continuous variables)

with cancer mortality among patients with diabetes is consistent with a previous cohort study reporting that total intake of n-3 PUFAs from diet and supplements was associated with lower cancer mortality in a general population. ${ }^{51}$ In addition, meta-analyses have concluded that n-3 PUFA intake was inversely related to risks of developing breast, ${ }^{52}{ }^{53}$ prostate, ${ }^{54}$ or liver cancer. ${ }^{55}$ In rodent models, n-3 PUFA supplements also ameliorated the diabetic phenotype and subsequently reduced the risk of colorectal cancer by inhibiting colonic expression of hepatocyte nuclear factor $4 \alpha$ and $\beta$-catenin/Tcf. ${ }^{56}$

Diets enriched with MUFAs, in comparison with carbohydrates, have been reported to improve metabolic risk factors in adults with type 2 diabetes. ${ }^{57}$ Consistently, substitution of MUFAs for carbohydrates was associated with lower total mortality among people with diabetes in the European Prospective Investigation into Cancer and Nutrition. ${ }^{58}$ We have recently reported that the associations of dietary MUFAs with risk of coronary heart disease were dependent on the food sources: MUFAs from plant foods were associated with a lower coronary heart disease risk, whereas their counterparts from animal sources were associated with a higher risk, possibly because of confounding by other food constitutes in animal produts. ${ }^{59}$ In the current study, we did not detect significant associations with total or cardiovascular disease mortality for total MUFAs or plant derived MUFAs, but animal based MUFAs were significantly associated with total mortality. Similarly, the Kuopio Ischemic Heart Disease Risk Factor study reported that dietary MUFAs, which were mainly from animal products in the study population, were associated with a higher risk of fatal coronary heart disease. ${ }^{60}$ Taken together, these data emphasize the role of food sources in determining the effects of MUFA intake on cardiovascular health in general populations and people with diabetes.

\section{Strengths and limitations}

This investigation has the strength of using the repeated assessments of diet after diagnosis of diabetes, which can help to capture potential dietary changes typically observed among people with type 2 diabetes. ${ }^{1258}$ Other strengths include the relatively large sample size, long term follow-up with a high follow-up rate (>90\%), and repeated measurements of other lifestyle factors. In addition, when we excluded participants who died within four years or those with high body mass index, the results did not change materially, indicating the robustness of our findings.

The study also has limitations. Firstly, glycemic control and severity of diabetes were not rigorously assessed. However, when we adjusted for duration of diabetes in the model and further adjusted for the use of insulin and hypoglycemic drugs, the results remained unchanged. Prevalent cases may differ from incident cases in terms of their risk profiles, but restricting the analysis to incident cases yielded similar results. Secondly, measurement errors in self reported diet and lifestyle factors were inevitable and may attenuate the associations of interest owing to the prospective study design. Thirdly, as in any observational study, we cannot exclude the role of residual confounding due to errors in measurement of covariates adjusted for in the analyses. In addition, we could not rule out unmeasured confounding by adherence to drug treatment, psychosocial stress, or other factors that were not assessed in the cohorts. 
Despite the shared sources of saturated fats and cholesterol, our findings are independent of dietary cholesterol intake, possibly because the effect of dietary cholesterols on circulating concentrations of cholesterol is relatively small. ${ }^{61}$ We also controlled for self reported hypercholesterolemia and use of lipid lowering drugs, and results did not change materially, suggesting that these factors may not fully account for the associations that we observed. Lastly, causality may not be established because of the observational nature of this study.

\section{Conclusions and implications}

Among US men and women with type 2 diabetes, dietary intake of PUFAs, especially linoleic acid and marine n-3 PUFAs, is associated with lower total and cardiovascular disease mortality. Our results suggest that dietary PUFAs, in replacement of saturated fatty acids or carbohydrates, may facilitate long term survival among adults with type 2 diabetes.

\section{AUTHOR AFFILIATIONS}

${ }^{1}$ Department of Nutrition, School of Public Health, Zhejiang

University School of Medicine, Hangzhou, Zhejiang, China

${ }^{2}$ Department of Nutrition, Harvard T.H. Chan School of Public Health, Boston, MA, USA

${ }^{3}$ CAS Key Laboratory of Nutrition, Metabolism and Food Safety, Institute of Nutrition and Health, Shanghai Institutes for Biological Sciences, University of Chinese Academy of Sciences, Chinese Academy of Sciences, Shanghai, China

${ }^{4}$ Division of General Internal Medicine, Division of Global Health Equity, Department of Medicine, Brigham and Women's Hospital, Department of Global Health and Social Medicine, Harvard Medical School, Boston, MA, USA

${ }^{5}$ Department of Epidemiology, Harvard T.H. Chan School of Public Health, Boston, MA, USA

${ }^{6}$ Channing Division of Network Medicine, Department of Medicine, Brigham and Women's Hospital and Harvard Medical School, Boston, MA, USA

${ }^{7}$ Division of Preventive Medicine, Department of Medicine, Brigham and Women's Hospital and Harvard Medical School, Boston, MA, USA

${ }^{8}$ Division of Women's Health, Department of Medicine, Brigham and Women's Hospital and Harvard Medical School, Boston, MA, USA

Contributors: JJ, GZ, and QS conceived and designed the study. JJ, GZ, $\mathrm{GL}$, and QS analyzed and interpreted data. JJ drafted the manuscript, and FBH, EBR, KMR, JEM, and QS revised it critically for important intellectual content. All authors provided final approval of the version to be published. The corresponding author attests that all listed authors meet authorship criteria and that no others meeting the criteria have been omitted. J) is the guarantor.

Funding: This study was sponsored by the National Institutes of Health (CA186107, CA167552, CA87969, DK082486, HL35464, DK058845, HL088521, and HL034594). GZ is supported by the 100 Talents Program of the Chinese Academy of Sciences, the National Key Research and Development Program of China (2018YFC604404), and the key deployment project of the Chinese Academy of Sciences (ZDBS-SSW-DQC-01). The funders had no role in the design and conduct of the study; collection, management, analysis, and interpretation of the data; preparation, review, and approval of the manuscript; or the decision to submit the manuscript for publication.

Competing interests: All authors have completed the ICMJE uniform disclosure form at www.icmje.org/coi_disclosure.pdf (available on request from the corresponding author) and declare: support from the National Institutes of Health for the submitted work; GZ is supported by a postdoctoral fellowship funded by Unilever R\&D, Vlaardingen, Netherlands; QS has received ad hoc consulting fees from Emavant Solutions $\mathrm{GmbH}$; FBH has been supported by grants HL60712, HL118264, and DK112940 from the National Institutes of Health and received research support from the California Walnut Commission, honorariums for lectures from Metagenics and Standard Process, and honorariums from Diet Quality Photo Navigation, outside the submitted work; no other relationships or activities that could appear to have influenced the submitted work.

Ethical approval: The study protocol was approved by the institutional review boards of the Brigham and Women's Hospital and the Harvard T.H. Chan School of Public Health. The completion of the self administered questionnaire was considered to imply informed consent.

Transparency declaration: The manuscript's guarantor affirms that the manuscript is an honest, accurate, and transparent account of the study being reported; that no important aspects of the study have been omitted; and that any discrepancies from the study as planned (and, if relevant, registered) have been explained.

Data sharing: Data can be shared through NHS and HPFS cohort data sharing policies that can be found at https://www.nurseshealthstudy. org/researchers.

This is an Open Access article distributed in accordance with the Creative Commons Attribution Non Commercial (CC BY-NC 4.0) license, which permits others to distribute, remix, adapt, build upon this work non-commercially, and license their derivative works on different terms, provided the original work is properly cited and the use is noncommercial. See: http://creativecommons.org/licenses/by-nc/4.0/.

1 NCD Risk Factor Collaboration (NCD-RisC). Worldwide trends in diabetes since 1980: a pooled analysis of 751 population-based studies with 4.4 million participants. Lancet 2016;387:1513-30. doi:10.1016/S0140-6736(16)00618-8

2 International Diabetes Federation. IDF diabetes atlas. 7th ed. IDF, 2015.

3 American Diabetes Association. 9. Cardiovascular disease and risk management. Diabetes Care 2017;40(Suppl 1):S75-87 doi:10.2337/dc17-S012

4 American Diabetes Association. 5. Lifestyle management: standards of medical care in diabetes-2019. Diabetes Care 2019;42(Suppl 1):S46-60. doi:10.2337/dc19-S005

5 Guasch-Ferré M, Hruby A, Toledo E, et al. Metabolomics in prediabetes and diabetes: a systematic review and meta-analysis. Diabetes Care 2016;39:833-46. doi:10.2337/dc15-2251

6 Seligman BG, Biolo A, Polanczyk CA, Gross JL, Clausell N. Increased plasma levels of endothelin 1 and von Willebrand factor in patients with type 2 diabetes and dyslipidemia. Diabetes Care 2000;23:1395-400. doi:10.2337/diacare.23.9.1395

7 Bowman L, Mafham M, Wallendszus K, et al, ASCEND Study Collaborative Group. Effects of $\mathrm{n}-3$ fatty acid supplements in diabetes mellitus. N Engl J Med 2018;379:1540-50. doi:10.1056/ NEJMoa1804989

8 Shai I, Schwarzfuchs D, Henkin Y, et al, Dietary Intervention Randomized Controlled Trial (DIRECT) Group. Weight loss with a low-carbohydrate, Mediterranean, or low-fat diet. N Engl) Med 2008.359.229-41 doi:10.1056/NEIMoa0708681

9 Brehm BJ, Lattin BL, Summer SS, et al. One-year comparison of a high-monounsaturated fat diet with a high-carbohydrate diet in type 2 diabetes. Diabetes Care 2009;32:215-20. doi:10.2337/ dc08-0687

10 Bloomfield HE, Koeller E, Greer N, MacDonald R, Kane R, Wilt T). Effects on health outcomes of a Mediterranean diet with no restriction on fat intake: a systematic review and meta-analysis. Ann Intern Med 2016;165:491-500. doi:10.7326/M16-0361

11 Estruch R, Ros E, Salas-Salvadó J, et al, PREDIMED Study Investigators. Primary prevention of cardiovascular disease with a mediterranean diet supplemented with extra-virgin olive oil or nuts. $N$ Engl J Med 2018;378:e34. doi:10.1056/NEJMoa1800389

12 dos Santos AL, Weiss T, Duarte CK, Gross IL, de Azevedo MJ, Zelmanovitz T. Dietary fat composition and cardiac events in patients with type 2 diabetes. Atherosclerosis 2014;236:31-8. doi:10.1016/j.atherosclerosis.2014.06.014

$13 \mathrm{Hu} F \mathrm{~B}, \mathrm{Cho}$ E, Rexrode KM, Albert CM, Manson JE. Fish and longchain $\omega$-3 fatty acid intake and risk of coronary heart disease and total mortality in diabetic women. Circulation 2003;107:1852-7. doi:10.1161/01.CIR.0000062644.42133.5F

14 Tanasescu M, Cho E, Manson JE, Hu FB. Dietary fat and cholesterol and the risk of cardiovascular disease among women with type 2 diabetes. Am J Clin Nutr 2004;79:999-1005. doi:10.1093/ ajcn/79.6.999

15 Patel YR, Gadiraju TV, Gaziano JM, Djoussé L. Adherence to healthy lifestyle factors and risk of death in men with diabetes mellitus: The Physicians' Health Study. Clin Nutr 2018;37:139-43. doi:10.1016/j. clnu.2016.11.003

16 Willett WC, Green A, Stampfer MJ, et al. Relative and absolute excess risks of coronary heart disease among women who smoke 
cigarettes. N Engl J Med 1987;317:1303-9. doi:10.1056/ NEJM198711193172102

17 Colditz GA, Rimm EB, Giovannucci E, Stampfer MJ, Rosner B, Willett WC. A prospective study of parental history of myocardial infarction and coronary artery disease in men. Am J Cardiol 1991;67:933-8. doi:10.1016/0002-9149(91)90163-F

18 Hu FB, Manson JE, Stampfer MJ, et al. Diet, lifestyle, and the risk of type 2 diabetes mellitus in women. N Engl J Med 2001;345:790-7. doi:10.1056/NEJMoa010492

19 Mozaffarian D, Hao T, Rimm EB, Willett WC, Hu FB. Changes in diet and lifestyle and long-term weight gain in women and men. N Engl J Med 2011:364:2392-404 doi:10.1056/NEIMoa1014296

20 National Diabetes Data Group. Classification and diagnosis of diabetes mellitus and other categories of glucose intolerance. Diabetes 1979;28:1039-57. doi:10.2337/diab.28.12.1039

21 American Diabetes Association. Standards of medical care in diabetes--2010. Diabetes Care 2010;33(Suppl 1):S11-61. doi:10.2337/dc10-S011

22 Manson JE, Rimm EB, Stampfer MJ, et al. Physical activity and incidence of non-insulin-dependent diabetes mellitus in women. Lancet 1991;338:774-8. doi:10.1016/0140-6736(91)90664-B

23 Hu FB, Leitzmann MF, Stampfer MJ, Colditz GA, Willett WC, Rimm EB. Physical activity and television watching in relation to risk for type 2 diabetes mellitus in men. Arch Intern Med 2001;161:1542-8. doi:10.1001/archinte.161.12.1542

24 Rimm EB, Giovannucci EL, Stampfer MJ, Colditz GA, Litin LB, Willett WC. Reproducibility and validity of an expanded self-administered semiquantitative food frequency questionnaire among male health professionals. Am J Epidemiol 1992;135:1114-26, discussion 1127 36. doi:10.1093/oxfordjournals.aje.a116211

25 Willett WC, Sampson L, Stampfer MJ, et al. Reproducibility and validity of a semiquantitative food frequency questionnaire. Am J Epidemiol 1985;122:51-65. doi:10.1093/oxfordjournals.aje. a114086

26 Hu FB, Stampfer MJ, Rimm E, et al. Dietary fat and coronary heart disease: a comparison of approaches for adjusting for total energy intake and modeling repeated dietary measurements. Am J Epidemiol 1999;149:531-40. doi:10.1093/oxfordjournals.aje. a009849

27 Willett WC, Lenart E. Reproducibility and validity of food-frequency questionnaires. In: Willett WC, ed. Nutritional epidemiology. 3rd ed. Oxford University, 2013

28 Wolf AM, Hunter DJ, Colditz GA, et al. Reproducibility and validity of a self-administered physical activity questionnaire. Int J Epidemiol 1994;23:991-9. doi:10.1093/ije/23.5.991

29 Hu FB, Willett WC, Li T, Stampfer MJ, Colditz GA, Manson JE. Adiposity as compared with physical activity in predicting mortality among women. N Engl J Med 2004;351:2694-703. doi:10.1056/ NEJMoa042135

30 Rich-Edwards JW, Corsano KA, Stampfer MJ. Test of the national death index and equifax nationwide death search. Am J Epidemiol 1994;140:1016-9. doi:10.1093/oxfordjournals.aje. a117191

31 Tobias DK, Pan A, Jackson CL, et al. Body-mass index and mortality among adults with incident type 2 diabetes. $N$ Engl $)$ Med 2014:370:233-44. doi:10.1056/NEIMoa1304501

32 Kulldorff M, Sinha R, Chow WH, Rothman N. Comparing odds ratios for nested subsets of dietary components. Int J Epidemiol 2000;29:1060-4. doi:10.1093/ije/29.6.1060

33 Guasch-Ferré M, Zong G, Willett WC, et al. Associations of monounsaturated fatty acids from plant and animal sources with total and cause-specific mortality in two US prospective cohort studies. Circ Res 2019;124:1266-75. doi:10.1161/ CIRCRESAHA.118.313996

34 Wang DD, Li Y, Chiuve SE, et al. Association of specific dietary fats with total and cause-specific mortality. JAMA Intern Med 2016:176:1134-45. doi:10.1001/jamainternmed.2016.2417

35 Ramsden CE, Zamora D, Leelarthaepin B, et al. Use of dietary linoleic acid for secondary prevention of coronary heart disease and death: evaluation of recovered data from the Sydney Diet Heart Study and updated meta-analysis. BMJ 2013;346:e8707. doi:10.1136/bmj. e8707

36 Chowdhury R, Warnakula S, Kunutsor S, et al. Association of dietary, circulating, and supplement fatty acids with coronary risk: a systematic review and meta-analysis. Ann Intern Med 2014:160:398-406. doi:10.7326/M13-1788

37 Sacks FM, Lichtenstein AH, Wu JHY, et al, American Heart Association. Dietary Fats and Cardiovascular Disease: A Presidential Advisory From the American Heart Association. Circulation 2017;136:e1-23. doi:10.1161/CIR 0000000000000510

38 Heine RJ, Mulder C, Popp-Snijders C, van der Meer J, van der Veen EA. Linoleic-acid-enriched diet: long-term effects on serum lipoprotein and apolipoprotein concentrations and insulin sensitivity in noninsulin-dependent diabetic patients. Am J Clin Nutr 1989;49:448-56. doi:10.1093/ajcn/49.3.448
39 Lee JH, O'Keefe JH, Lavie CJ, Harris WS. Omega-3 fatty acids: cardiovascular benefits, sources and sustainability. Nat Rev Cardiol 2009:6:753-8. doi:10.1038/nrcardio.2009.188

40 Saravanan P, Davidson NC, Schmidt EB, Calder PC. Cardiovascular effects of marine omega-3 fatty acids. Lancet 2010;376:540-50. doi:10.1016/S0140-6736(10)60445-X

41 Aung T, Halsey J, Kromhout D, et al, Omega-3 Treatment Trialists' Collaboration. Associations of omega-3 fatty acid supplement use with cardiovascular disease risks: Meta-analysis of 10 trials involving 77917 individuals. JAMA Cardiol 2018;3:225-34. doi:10.1001/ jamacardio.2017.5205

42 Siscovick DS, Barringer TA, Fretts AM, et al, American Heart Association Nutrition Committee of the Council on Lifestyle and Cardiometabolic Health; Council on Epidemiology and Prevention; Council on Cardiovascular Disease in the Young; Council on Cardiovascular and Stroke Nursing; and Council on Clinical Cardiology. Omega-3 Polyunsaturated Fatty Acid (Fish Oil) Supplementation and the Prevention of Clinical Cardiovascular Disease: A Science Advisory From the American Heart Association. Circulation 2017;135:e867-84. doi:10.1161/ CIR.0000000000000482

43 Manson JE, Cook NR, Lee IM, et al, VITAL Research Group. Marine $n-3$ fatty acids and prevention of cardiovascular disease and cancer. N Engl J Med 2019;380:23-32. doi:10.1056/ NEJMoa1811403

44 Bhatt DL, Steg PG, Miller M, et al, REDUCE-IT Investigators. Cardiovascular risk reduction with icosapent ethyl for hypertriglyceridemia. N Engl J Med 2019;380:11-22. doi:10.1056/ NEJMoa1812792

45 Tajuddin N, Shaikh A, Hassan A. Prescription omega-3 fatty acid products: considerations for patients with diabetes mellitus. Diabetes Metab Syndr Obes 2016;9:109-18.

46 De Luis DA, Conde R, Aller R, et al. Effect of omega-3 fatty acids on cardiovascular risk factors in patients with type 2 diabetes mellitus and hypertriglyceridemia: an open study. Eur Rev Med Pharmacol Sci 2009; 13:51-5.

47 Hendrich S. (n-3) fatty acids: clinical trials in people with type 2 diabetes. Adv Nutr 2010;1:3-7. doi:10.3945/an.110.1003

48 Oikawa S, Yokoyama M, Origasa H, et al, JELIS Investigators, Japan. Suppressive effect of EPA on the incidence of coronary events in hypercholesterolemia with impaired glucose metabolism: Sub-analysis of the Japan EPA Lipid Intervention Study (JELIS). Atherosclerosis 2009;206:535-9. doi:10.1016/j. atherosclerosis.2009.03.029

49 Bosch J, Gerstein HC, Dagenais GR, et al, ORIGIN Trial Investigators n-3 fatty acids and cardiovascular outcomes in patients with dysglycemia. N Engl J Med 2012;367:309-18. doi:10.1056/ NEJMoa1203859

50 Szablewski L. Diabetes mellitus: influences on cancer risk. Diabetes Metab Res Rev 2014:30:543-53. doi:10.1002/dmrr.2573

51 Bell GA, Kantor ED, Lampe JW, Kristal AR, Heckbert SR, White E. Intake of long-chain $\omega-3$ fatty acids from diet and supplements in relation to mortality. Am J Epidemiol 2014;179:710-20. doi:10.1093/aje/ kwt326

52 Zheng J-S, Hu X-J, Zhao Y-M, Yang J, Li D. Intake of fish and marine n-3 polyunsaturated fatty acids and risk of breast cancer: metaanalysis of data from 21 independent prospective cohort studies. BMJ 2013;346:f3706. doi:10.1136/bmj.f3706

53 Liu J, Ma DWL. The role of n-3 polyunsaturated fatty acids in the prevention and treatment of breast cancer. Nutrients 2014;6:5184223. doi:10.3390/nu6115184

54 Fu Y-Q, Zheng J-S, Yang B, Li D. Effect of individual omega-3 fatty acids on the risk of prostate cancer: a systematic review and dose-response meta-analysis of prospective cohort studies. J Epidemiol 2015;25:261-74. doi:10.2188/jea.JE20140120

55 Gao M. Sun K, Guo M, et al. Fish consumption and n-3 polyunsaturated fatty acids, and risk of hepatocellular carcinoma: systematic review and meta-analysis. Cancer Causes Control 2015:26:367-76. doi:10.1007/s10552-014-0512-1

56 Algamas-Dimantov A, Yehuda-Shnaidman E, Hertz R, Peri I, Bar-Tana J, Schwartz B. Prevention of diabetes-promoted colorectal cancer by $(n-3)$ polyunsaturated fatty acids and (n-3) PUFA mimetic. Oncotarget 2014;5:9851-63. doi:10.18632 oncotarget.2453

57 Qian F, Korat AA, Malik V, Hu FB. Metabolic Effects of monounsaturated fatty acid-enriched diets compared with carbohydrate or polyunsaturated fatty acid-enriched diets in patients with type 2 diabetes: a systematic review and meta-analysis of randomized controlled trials. Diabetes Care 2016;39:1448-57. doi:10.2337/dc16-0513

58 Campmans-Kuijpers MJ, Sluijs I, Nöthlings U, et al. The association of substituting carbohydrates with total fat and different types of fatty acids with mortality and weight change among diabetes patients. Clin Nutr 2016;35:1096-102. doi:10.1016/j. clnu.2015.08.003 
59 Zong G, Li Y, Sampson L, et al. Monounsaturated fats from plant and animal sources in relation to risk of coronary heart disease among US men and women. Am J Clin Nutr 2018;107:445-53. doi:10.1093/ ajcn/nqx004

60 Virtanen JK, Mursu J, Tuomainen TP, Voutilainen S. Dietary fatty acids and risk of coronary heart disease in men: the Kuopio Ischemic Heart Disease Risk Factor Study.
Arterioscler Thromb Vasc Biol 2014;34:2679-87. doi:10.1161/ ATVBAHA.114.304082

61 McNamara DJ. Dietary cholesterol, heart disease risk and cognitive dissonance. Proc Nutr Soc 2014;73:161-6. doi:10.1017/ S0029665113003844

\section{Supplementary materials}

